\title{
Tangence
}

\section{LE TEMPS D'APRÈS l'histoire et le postmodernisme}

\section{Pierre Ouellet}

Numéro 39, mars 1993

La fiction postmoderne

URI : https://id.erudit.org/iderudit/025757ar

DOI : https://doi.org/10.7202/025757ar

Aller au sommaire du numéro

Éditeur(s)

Tangence

ISSN

0226-9554 (imprimé)

1710-0305 (numérique)

Découvrir la revue

Citer cet article

Ouellet, P. (1993). LE TEMPS D'APRÈS l'histoire et le postmodernisme. Tangence, (39), 112-131. https://doi.org/10.7202/025757ar d'utilisation que vous pouvez consulter en ligne.

https://apropos.erudit.org/fr/usagers/politique-dutilisation/ 


\section{LE TEMPS D'APRÈs l'histoire et le postmodernisme}

\section{Pierre Ouellet}

L'histoire se réinvente sans cesse. On n'est pas au bout des Grands Récits, dont Jean-François Lyotard nous annonçait la mort il y a une quinzaine d'années. L'histoire se fait, une fois pour toutes, mais se récrit au gré des ans, des décennies. Et on dirait qu'elle se récrie, furieuse qu'on dise d'elle aujourd'hui le contraire de ce qu'on en pourrait dire demain. Les faits sont malléables, terre glaise dont on fabrique les fictions, les artefacts, comme le potier l'amphore, donnant à chaque fois une forme différente à son tas de boue. Les faits historiques n'y échappent pas. Ce sont des "montres molles", qu'il faut à tout instant remettre à l'heure. Sous la rigidité mécanique des rouages qui en meuvent les aiguilles avec la régularité du métronome, l'extrême mollesse du temps qui coule, s'écoule, non comme le fleuve de l'Éphésien, mais comme le plâtre dans quelque moule, où il ne durcit pas sans se faire friable, fragile, voué à la ruine, qu'un autre plâtre fortifiera, ou remplacera, prothèse parmi les membres dispersés d'une historicité qu'on n'aperçoit qu'en miettes.

Il est arrivé à Clio ce qui jadis frappa Orphée: les Ménades l'ont démembrée. Il faut en recoller les morceaux - membra disjecta que l'on raboute, puzzle du Grand Corps collectif dont on ne sait plus, bientôt, mettre la tête sur les épaules et tout le squelette debout. Les Grands Récits ne sont pas morts, ils ont seulement changé. Il ne content plus l'histoire par le commencement, perdu dans la nuit des temps, mais par la fin, souvent, ou par le milieu, collant bout à bout bras et jambes, coudes et genoux, les yeux dans le dos et le cœur au milieu du visage. Collage et recollage, l'Histoire n'apparaît plus que sous les multiples formes où ses morceaux s'agencent: dans l'ordre et le désordre. De nouveaux histrions voient le jour, qui se prennent pour des historiens, changeant l'ordre non des mots dans la phrase comme Monsieur Jourdain mais des faits dans leur chronologie, donnant ainsi l'illusion de faire non tant de la prose que de l'historiographie. On a raconté l'Histoire comme l'Épopée du progrès, le Sujet, tel Rastignac ou Sorel, allant droit devant soi, sans retour ni recul, fonçant, arriviste comme est la Science ou la Technique, le Capital 
ou l'Industrie - le héros Ego et l'héroïne Idée marchant en éclaireur, devant la masse des êtres et des faits, qu'ils guident dans le même Sens, tous au pas, que scande un seul et même Rythme. Puis, comme on se lasse de tout, et de Balzac comme de Stendhal, on l'a racontée autrement, changeant le nom des personnages, Moderne devenu Postmoderne, et leur date de naissance, les siècles et les décennies se chevauchant ou se superposant. On l'a contée à la Borges, creusant partout des labyrinthes, où le sens se perd et les voies se multiplient, pluralisant les points de vue - enrichissement de la vision où l'appauvrissement du sens s'oublie.

L'histoire est palimpseste, certes, chacun de ses récits effaçant le précédent, mais palinodie aussi, chacun contredit par le suivant, qui est volte-face, rétractation, revirement. Le Postmodernisme n'est pas seulement la théorie de la postmodernité - ou la Critique de la Modernité - , il est, bien plus fondamentalement, la redéfinition même de l'historicité. Non pas de l'historicité de l'art ou de la littérature, sur quoi il s'est longuement penché, ni non plus de la seule historicité du Sujet, qu'il a contribué à mettre au jour, exposant ses zones d'ombre, mais de l'historicité de l'Histoire elle-même, qui va maintenant dans tous les sens, qu'aucune orientation privilégiée ne permet de limiter. On a déchronologisé l'Histoire, dit-on, et ce non seulement dans la façon dont l'art et la littérature dits postmodernes remettent à jour et en jeu leur propre historicité, mais dans la manière même dont on refait l'histoire des Arts ou des Lettres et l'Histoire tout court. En peinture et en architecture, comme en littérature bientôt, le postmodernisme n'aura été qu'un moment de l'histoire, après et avant tant d'autres, dont on commence maintenant à pouvoir faire le tour, cernant le mouvement de pensée qui lui a donné naissance et qu'on voit d'ores et déjà mourir de sa belle mort: le kitsch saisi par le kitsch, le mauvais goût même devenu de mauvais goût. L'immeuble ou le tableau postmodernes, partout copiés, imités, caricaturés, déclenchent le rire et la moquerie, Joconde à qui l'on met non tant de modernes moustaches à la Duchamp qu'un large bonnet en forme de chapiteau corinthien et des pendants d'oreille en forme de colonnes doriques. Bref, la manière dont l'architecture et la peinture ont remis à l'honneur les formes ornementales du passé - violemment critiquées par notre Modernité fonctionaliste, utilitariste, minimaliste et réductionniste, dès le début de ce siècle - est à son tour chose $d u$ passé, laissant de la sorte deviner le rôle qu'elle a pu jouer dans l'histoire récente des mouvements artistiques, sur lesquels il 
114

semble que son influence ne s'exerce plus guère que de façon fort détournée.

Il en va autrement de la littérature, dont l'histoire n'obéit pas à la même clôture: les contours d'un mouvement littéraire postmoderne sont loin d'être aussi précis. Si le postmodernisme architectural et pictural - musical aussi - définit un courant artistique international dont l'émergence remonte au milieu des années soixante-dix et dont la progressive disparition marque la fin des années quatre-vingt, il semble que la littérature postmoderne n'ait été identifiée comme telle et n'ait fait l'objet d'une appropriation par les écrivains eux-mêmes qu'au sein de la culture anglo-américaine, où l'on a tenté de nombreuses repériodisations, qui font remonter la postmodernité littéraire à Vladimir Nabokov quand ce n'est pas à William Faulkner, et qui étendent son influence jusque sur la littérature la plus contemporaine, couvrant ainsi plus de cinquante ans d'histoire. En passant des Beaux-Arts aux Belles-Lettres, la Postmodernité a gagné en profondeur temporelle ou historique ce qu'elle a perdu en étendue territoriale ou géographique: mieux circonscrite dans une littérature nationale, l'anglo-américaine, qui n'a cessé, toutefois, d'essaimer dans l'ensemble des sociétés industrielles où elle exerce son ascendant, elle reste difficilement cernable dans le temps, même si l'on s'entend pour en situer l'épicentre dans l'œuvre des Barth, Barthelme, Coover, Hawks, Pynchon et autres Vonnegut, qui s'étale déjà sur plus de trois décennies. Le phénomène n'est donc pas tout à fait comparable à ce qui s'est passé dans les Arts. On a ainsi beaucoup de peine à coller l'étiquette postmoderne à l'un ou l'autre des écrivains francophones importants des deux dernières décennies. De même, Octavio Paz a récemment fait remarquer le ridicule qu'il y avait à importer cette étiquette dans la littérature hispanique et latino-américaine; fustigeant l'utilisation que la critique américaine a jadis pu faire du concept de modernisme, il montre que l'usage de l'expression "postmodernisme" obéit sensiblement à la même règle:

[...] appeler modernism un courant de langue anglaise apparu trente ans après le nôtre ['hispanique ${ }^{1}$, est un signe d'arrogance

1 Paz évoque ici l'avènement du modernisme en langue espagnole, avec des auteurs comme Rubén Dario, Juan Ramon Jiménez, José Marti ou 
culturelle, d'ethnocentrisme et d'insensibilité historique. On peut en dire autant de l'expression postmodernism, utilisée pour désigner de nouvelles sensibilités artistiques et littéraires aux États-Unis ou ailleurs. Le plus triste - ou le plus risible - est que ces termes, avec le sens particulier que leur donnent les Anglo-Américains commencent à trouver un écho non seulement dans divers pays européens, mais en Amérique latine comme en Espagne. Cette mise au point n'a rien d'oiseux et ne reflète aucun nationalisme attardé: la controverse autour du modernism let du postmodernism] n'est pas une querelle de mots, mais de signifiés, de concepts et d'histoire ${ }^{2}$.

Plus récemment encore, Jean-Marie Gleize soulignait le fait qu'en France l'expression "postmoderne", loin de désigner un courant "nouveau ", "révolutionnaire "ou "progressiste" en littérature, comme ce fut le cas aux États-Unis dans les dernières décennies, n'en déplaise aux partisans de la "fin de l'histoire" et de la neutralisation des Idéologies ${ }^{3}$, dénotait un type mal défini de "réaction " marquée par tous les "retour à ", qui ont donné lieu à de nombreux manifestes travestis en "anthologies" comme celle qu'on trouve dans le numéro 443 de la $N R F$ sur les "Poètes des années quatre-vingt "4: "Néolyrisme, néoréalisme, néoromantisme, néosymbolisme, néoreligiosité, tous ces poètes dessinent les contours d'un "postmodernisme" caractérisé (c'est-à-dire d'un antimodernisme virulent) ${ }^{5}$, nous rappelle Gleize. Si ces réflexions, avec celles de Paz, concernent plus directement la poésie, il ne serait certes pas trop difficile de démontrer que le phénomène s'ap-

Antonio Machado, qui ont précédé les Pound, Eliot, Williams et Hemingway, de la même manière que les Rimbaud, Mallarmé et Proust auront de plus loin encore annoncé l'émergence du modernisme littéraire occidental.

2 Octavio Paz (1992 [1990]), L'autre voix. Poésie et fin de siècle, trad. par J.C. Masson, Paris, Gallimard, coll. *Arcades *, p. 66-67.

3 Paz dit encore: «Se baptiser postmoderne [pour les Américains, notammentl est une manière somme toute ingénue d'affirmer que l'on est très moderne. [...] S'appeler postmoderne revient à être encore et toujours prisonnier du temps successif, linéaire et progressif * (op. cit., p. 65).

4 Paru en décembre 1980, ce numéro * programmatique * de la NRF réunissait des ceuvres de $M$. Calonne, J.-P. Chambon, J.-N. Chrisment, Ph. Delaveau, G. Goffette, H. Kadour, J.-P. Lemaire et P. de Roux, qui constituent depuis lors l'essentiel de ce que publie la collection de poésie de chez Gallimard.

5 Jean-Marie Gleize (1992), A noir. Poésie et littéralité, Paris, Seuil, coll. *Fiction \& Cie *, p. 148, note 1. 
116

plique aussi bien et peut-être mieux encore au roman, comme en témoignent les numéros spéciaux de certaines revues comme Rive droite, par exemple, dont la "Nouveauté" affichée réside dans une critique non pas tant du Nouveau en soi, comme ce fut le cas en art visuel, que des innovations formelles de la narration romanesque, en France, depuis le début des années cinquante jusqu'au milieu des années soixante-dix ${ }^{6}$. On voit que la notion de postmodernisme en littérature est loin d'être homogène, dès lors qu'on s'avise de traverser les frontières géographiques, et qu'elle ne l'est guère plus lorsqu'on se met à observer les différentes bornes historiques que lui confèrent les multiples datations et périodisations contradictoires tentées par la critique américaine depuis plus d'une dizaine d'années.

Le discours critique qui a accompagné ou suivi de près l'émergence et l'évolution de ce courant littéraire aux États-Unis et, dans une moindre mesure, au Canada anglais, n'est pas non plus du même ordre que celui qui, dans une même volonté de "dépassement" de la Modernité par une sorte de ressac historique où refluerait une à une chaque nouvelle vague que l'Histoire a connue, n'a pas manqué de critiquer non tant les Grands Récits sur lesquels la pensée moderne s'est édifiée que les Maîtres à penser qui, dans les années soixante et soixante-dix surtout, auraient largement contribué à nous «édifier» dans le sens de cette Modernité tant décriée. Ces Grands Maîtres, dont les noms ont pratiquement disparu du discours critique contemporain dans le monde francophone, où on ne les cite plus que dans une perspective exégétique ou historique, sinon hagiographique, comme c'est le cas pour certains d'entre eux, constituent pourtant des références obligées de la pensée postmoderne anglo-américaine, où les Althusser, Derrida, Foucault et Lacan ne cessent d'alimenter la réflexion critique ${ }^{7}$. De même, les grands mouvements

6 Il s'agit là essentiellement d'une réaction au Nouveau Roman, à Tel Quel et aux différentes Avant-gardes qui ont marqué un quart de siècle de vie littéraire en France quelques années après la dernière Guerre, réaction qui s'est manifestée entre autres par un retour au "romanesque*, à l'intrigue, à la linéarité du récit, à la psychologie des personnages, etc., qui semble tout le contraire de ce qu'ont tenté les écrivains angloaméricains identifiés au postmodernisme, comme on va le voir plus loin.

7 Althusser est devenu en France un objet historique: on publie son journal, sa correspondance, etc., tandis qu'on organise des colloques sur 
réformistes comme le féminisme, le postcolonialisme, la défense des minorités sexuelles ou ethniques, etc., qui dominent dans la critique américaine actuelle, constituent-ils l'une des légitimations les plus efficientes du postmodernisme littéraire et de ses prolongements philosophiques ou socio-politiques. Rien d'équivalent n'a marqué la critique de la modernité dans le monde francophone, où les écrivains soi-disant postmodernes, partisans des nouveaux "retours à n, n'ont trouvé d'alliés objectifs que chez les détracteurs de ce qu'on appelle de manière caricaturale la pensée soixante-huitarde, où sont pourtant nés et se sont développés les courants d'idées sur lesquels la critique postmoderniste américaine continue de s'appuyer.

La littérature française d' "après-la-modernité" n'est toutefois pas très homogène et plusieurs auteurs qui ont jadis pratiqué une forme de modernisme littéraire s'en sont démarqués sur des bases qui ne sont pas nécessairement "réactionnaires". Sollers passe de $H$ et Paradis à Femmes et Portrait du joueur, Sallenave, du Voyage d'Amsterdam et des Portes de Gubbio à La vie fantôme et aux Conversations conjugales, Quignard, du Lecteur et de Carus au Salon du Wurtemberg et aux Escaliers de Chambord, laissant entendre que l'expérimentation langagière et narrative, caractéristique de leurs premières ouvres, serait brusquement devenue obsolète, remplacée par une maîtrise des codes plus traditionnels de la fiction romanesque, sans qu'aucun discours justificateur, "réactionnaire" non plus que "révolutionnaire", n'ait marqué l'avènement de ces mutations, parfois réversibles d'ailleurs, comme en témoignent les exigences formelles des récentes œuvres d'un Pascal Quignard. De même, on ne peut soupçonner la Duras de L'amant, le Robbe-Grillet du Miroir qui revient ou la Sarraute d'Enfance d'avoir adopté une forme d'autobiographie, au demeurant fort peu traditionnelle, pour se démarquer de manière "réactionnelle" du modernisme qui caractérise leurs premières périodes. Il reste bien sûr à s'interroger sur ces changements de cap - pour les trois premiers auteurs cités, notamment, les trois derniers n'ayant pas franchement rompu avec les formes

la pensée de Lacan ou de Derrida et qu'on publie des biographies ou des témoignages qui les concernent, eux et leur époque. Leurs cuvres font désormais l'objet de commentaires philologiques, après avoir subi le feu nourri des critiques de la Nouvelle philosophie ou du nouveau rationalisme *, et elles ne peuvent plus être sérieusement reçues et utilisées comme discours d'autorité. 
d'énonciation et de narration qui les ont toujours caractérisés mais cette interrogation ne doit pas nécessairement prendre sa source dans les grands principes qui règlent la critique postmoderniste d'inspiration anglo-américaine.

L'utilisation des expressions "postmoderne ", "postmodernité " et "postmodernisme " relève souvent d'un très large malentendu, dont la polysémie du terme est en grande partie responsable. On peut à mon sens distinguer quatre usages fort différents de ces termes, que la Critique actuelle se plaît à croiser, produisant des effets de discours parfaitement "postmodernes" au sens péjoratif de l'expression, qui désigne aussi, sarcastiquement, quelque voisinage malheureux ou collage incongru. Un premier sens, très large, définit le terme à l'intérieur du discours philosophique et, plus particulièrement, de l'histoire de la philosophie: il décrit le passage d'une philosophie du Concept et du Sujet, marquant la modernité philosophique, de Descartes et Locke à Hegel et Husserl, support de l'Humanisme et de la Métaphysique occidentale, à une philosophie axée sur la déconstruction de la rationalité et de la subjectivité, amorcée par Nietzsche et poursuivie de manière plus ou moins divergente par Freud et Heidegger jusqu'à Lacan et Derrida, dont le but ultime est à chaque fois de "sortir " du discours métaphysique que le Cogito cartésien incarne dans la philosophie moderne. Cette sortie-là peut à bon droit prendre le nom de "pensée postmoderne ", dans la mesure où elle n'est possible que dans l'après-coup d'une Modernité qui a pris naissance d'une rupture d'avec la pensée scolastique et son interprétation des philosophies de l'Antiquité.

Une autre acception, assez large elle aussi, prend sa source dans le discours des sciences sociales (de la sociologie aux sciences politiques et économiques en passant par l'histoire); elle décrit le triple passage 1) des sociêtés dites industrielles, basées sur la production des biens matériels, aux sociétés dites postindustrielles, où les réseaux de communication et d'information dominent largement le champ de la production 2) des États colonialistes et impérialistes, marqués par l'ethnocentrisme européano-américain, à la multiplication des États postcolonialistes et à l'avènement des sociétés multiethniques ou des communautés dites transculturelles, et 3) des grandes Idéologies antagonistes (communisme et capitalisme) à un nouvel Ordre mondial qui ne s'accompagne plus que des différents discours alternatifs internes au Libéralisme politique et économique. Ces 
mutations socio-politico-économiques, sur fond de grands changements technologiques, peuvent donner lieu elles aussi à l'utilisation de l'étiquette "postmoderne" pour décrire le nouvel état du monde qu'elles ont engendré.

Une autre conception encore renvoie à l'usage que j'ai défini plus haut et qui décrit de manière beaucoup plus restreinte les changements survenus dans les pratiques artistiques vers la fin des années soixante-dix, en réaction contre le réductionisme minimaliste qui a marqué l'évolution de l'Art moderne depuis Cézanne, Matisse et Picasso, radicalisés ensuite par Kandinsky, Mondrian et Malévitch, dont le "Carré blanc sur fond blanc" n'a cessé de travailler de l'intérieur la modernité picturale jusqu'à Riemann, Marden, Mangold ou Lewit, qui ont dominé le monde de l'Art dès le milieu des années soixante. Cette contestation du réductionisme pictural est à peu près contemporaine des remises en cause du style international en architecture, où le fonctionalisme dominait déjà depuis plusieurs décennies. Cette mise en question des codes esthétiques de la modernité s'est bien sûr répandue assez vite dans les autres formes d'art, notamment en musique, où le sérialisme, l'atonalité, le dodécaphonisme et les différentes formes de pointillismes, équivalents, grosso modo, du formalisme pictural, ont commencé dès la même époque à perdre du terrain au profit d'une écriture musicale où la tonalité, notamment, revenait en force. Ces mutations prirent très tôt le nom de postmodernisme, indépendamment, souvent, des autres acceptions, socio-économiques ou philosophiques, de l'expression. Enfin, le dernier usage sur lequel j'aimerais m'attarder, puisqu'il constitue le noud des questions que je pose ici, est celui qui a présentement cours en littérature et dans la critique comme dans la théorie littéraires, dont certains courants s'appuient notamment sur un amalgame des trois premières définitions pour circonscrire l'objet d'étude et de réflexion qu'ils se sont donné ou, plus justement, qu'ils se sont construit.

Il faut d'abord distinguer un postmodernisme proprement littéraire, revendiqué par les auteurs, surtout les romanciers, depuis que John Barth, notamment, en a prophétiquement annoncé l'émergence dans les années soixante ${ }^{8}$, et une critique ou

8 On sait que Barth, dans son article sur "La littérature du renouvellement. La fiction postmoderniste. (Poétique, n*48, 1981, p. 395-405), panu origi- 
une théorie du postmodernisme qui, dans les années quatrevingt, a tenté de saisir et décrire rétrospectivement les mutations que la littérature - américaine, surtout, je le répète - avait connues depuis déjà une vingtaine d'années et même plus, selon que l'on fait remonter à tel ou tel auteur l'émergence d'un soidisant "style" ou d'une supposée "attitude" postmoderniste. S'il n'est pas contestable que quelque chose d'important se soit produit dans les Lettres, aux États-Unis, avec l'œuvre novatrice des Pynchon et des Vonnegut, il n'est pas du tout certain que, quoi qu'en dise John Barth, ces mutations soient proprement dues à une forme de postmodernisme ou soient tout au moins descriptibles en termes d'émergence d'une véritable postmodernité littéraire. Surtout lorsqu'on examine de près les caractéristiques que la critique dégage depuis une dizaine d'années des œuvres qui selon elle relèvent d'une esthétique postmoderne, comme l'usage massif de l'ironie, de la parodie, de l'allégorie, par le mélange des genres nobles et populaires, la subversion des canons littéraires, le collage, le recyclage et la citation plus ou moins déformée, le brouillage des genres (au double sens de "formes fixes " et de Genders) et des différents attributs sociaux et psychologiques de la subjectivité par une mise en cause du personnage monologique et des dramatis personnae, le télescopage des référents spatio-temporels par la mise en question de la linéarité et de la progressivité de l'Histoire ou par la déchronologisation du temps historique tel qu'il se dépose dans les différents styles d'époques diverses et les différentes écoles esthétiques, dont on mime ou copie, jusqu'à la grimace et la mimique, les traits les plus voyants, en les mélangeant outrancièrement, au risque d'afficher le pire des "mauvais goûts", la déstabilisation des valeurs morales héritées du puritanisme protestant par la mise en scène généralisée de la provocation ou la mise en valeur des minorités de tous ordres contre un ordre éthique dominant, identifiable à un ethno-phallo-logo-centrisme, que l'inquiétude et le doute généralisés dans lesquels le roman postmoderne est censé nous plonger contribueraient à mettre à mal de manière radicale, en une sorte d'activisme ou de militantisme du second degré, etc. Toutes ces caractéristiques, sans

nalement en janvier 1980 dans The Atlantic, rebaptisa rétrospectivement "postmoderniste " la clittérature de l'épuisement " qu'il avait identifiée et définie près de quinze ans plus tôt dans un article de la même revue, paru en août 1967. 
nul doute présentes dans la littérature américaine des dernières années, comme dans bien d'autres d'ailleurs, marquent sans conteste un changement notable dans l'esthétique romanesque de nos voisins du Sud, mais rien n'est moins sûr que la conclusion à laquelle en arrive la critique quand elle les attribue en bloc au "paradigme postmoderniste", dans la mesure où, comme on l'aura certainement remarqué, plusieurs de ces "traits "définissent d'ores et déjà la production romanesque et poétique de ce que la critique des années soixante et soixante-dix, notamment, appelait avec peut-être un excès de candeur la Modernité littéraire, faisant par là référence à Kafka, Rilke ou Musil, Joyce, Woolf ou Eliot, Mallarmé, Proust ou Céline, comme à Duras et Robbe-Grillet, Bernhard et Handke, Goyen et Gaddis, dignes ancêtres ou grands contemporains des Hawks, Coover et autres Barthelme. C'est sans doute pourquoi plusieurs critiques américains, comme le signale à juste titre John Barth ${ }^{9}$, considèrent le postmodernisme littéraire non comme une rupture d'avec le modernisme mais comme son développement, jusqu'à la caricature, les détracteurs du modernisme ne manquant pas, dès lors, d'attaquer du même coup son digne successeur.

Derrière toute tentative d'identification d'un style, d'une école, d'une attitude, d'un mouvement esthétique ou d'un courant de pensée supposé avoir marqué un changement important dans l'histoire d'une formation discursive donnée, se profile le problème fondamental de la datation et de la périodisation qui permettent seules d'ancrer l'innovation formelle ou thématique dans son historicité. Or il semble que, si le postmodernisme proprement littéraire s'est voulu ni plus ni moins qu'une poétique, dont on commence d'ailleurs à apercevoir aujourd'hui la clôture, ses effets esthétiques s'étant plus ou moins épuisés par saturation ou édulcoration, la critique postmoderniste n'a cessé, quant à elle, d'ancrer ce mouvement dans une histoire beaucoup plus large, qui oscille entre l'Histoire générale des idées - plus particulièrement celle de la pensée philosophique - et l'Histoire sociale et politique des quarante dernières années - plus spécifiquement celle de l'Après-guerre. Elle dépasse ainsi largement les ambitions de la critique d'art, qui n'a jamais eu d'autres 
buts, malgré ses velléités de mise en cause des notions modernistes de progrès et d'avant-garde, que de situer l'art dit postmoderne par rapport au "formalisme " et au "réductionnisme" qui l'ont immédiatement précédé, sans présupposer, par exemple, que cette esthétique pouvait rétrospectivement caractériser l'œuvre d'artistes plus anciens ni qu'elle pouvait représenter une quelconque fin de l'histoire de l'Art, celle-ci s'étant par ailleurs "vengée" de manière préventive d'une telle menace en annonçant il y a peu la fin même du postmodernisme artistique, le néomodernisme ayant vite pris sa place dès la fin des années quatrevingt, notamment par le biais du néocontructivisme en architecture. La portée que la critique littéraire a voulu donner à la littérature dite postmoderne déborde largement le cadre esthétique; on a voulu évaluer son impact sur les plans à la fois philosophique et sociopolitique, amalgamant à la poétique postmoderniste des conceptions et des acceptions de la "postmodernité "qui lui sont pour une grande part étrangères ou qui appartiennent à une toute autre temporalité ou à une historicité définissant d'autres formations discursives sinon d'autres ordres de réalité. Ce qui ne veut pas dire, bien sûr, qu'aucun lien n'existe entre les changements épistémiques dans le discours philosophique, d'une part, les mutations politico-économiques dans le champ social et historique, d'autre part, et les métamorphoses esthétiques plus ou moins brusques que connaissent les pratiques artistiques et littéraires. Cela signifie seulement qu'on ne peut confondre les différentes "lignes de temps" qui régissent l'historicité propre à chaque institution sociale, littérature comprise, sous peine de construire un concept d'Histoire à une seule vitesse, où tout irait dans le même sens et au même rythme, sous la baguette d'un seul et même chef, l'Idée ou le Progrès, ordonnateur suprême des Grands récits ou des Grandes symphonies du Temps, dont on a pourtant tout fait pour se débarrasser, préférant au fit historique les feuilletés d'histoires multiples et parfois contradictoires, même si toutes tiennent l'une sur l'autre, séparées seulement par un peu de jeu, où réside précisément la liberté qu'incarnent les Institutions humaines les plus fondamentales (langues, religions, arts, littératures et Droit) par rapport aux différents déterminismes historiques que les sciences économiques et sociales ont su mettre au jour.

Les ruptures esthétiques, épistémologiques ou socioéconomiques ne sont pas monnaie courante. Leur rareté seule leur donne une valeur dans l'histoire, à défaut de quoi elles 
seraient "normales" et ne déclencheraient aucun "Gestalt Shift" comme dit Thomas Kuhn, les changements de points de vue ne pouvant survenir que de manière discontinue, sans quoi ils ne sont pas "aperçus" et n'existent tout simplement pas. La continuité des faits, qui définit l'histoire dans son événementialité première, doit par conséquent faire l'objet d'une "perception seconde" où apparaisse ce que j'appellerais son "aspectualité " au sens linguistique du terme - soit le point de vue à partir duquel et l'intervalle ouvert ou fermé à l'intérieur duquel l'événement historique est perçu - pour que l'histoire puisse être considérée sous l'angle de la rupture, de l'innovation ou d'un quelconque changement profond, cela même si certaines dates semblent indiquer d'elles-mêmes une forme de discontinuité, laissant supposer que celle-ci s'appréhende en-deçà de nos représentations historiques, au sein même du flux événementiel ${ }^{10}$. La perception du discontinu dans l'ordre historique implique deux types de positions perceptives, sur lesquelles s'appuie toute discursivation des formes événementielles de la diachronie. La première position est celle du sujet observateur, énonciateur du discours historique, dont la situation définit la perspective dans laquelle l'événement sera vu, tandis que l'autre situe plus proprement l'objet, observé et mis en discours, à l'intérieur d'un intervalle temporel défini par une échelle de grandeurs où varient presque à l'infini les portions de temps historiques envisagées. Ainsi la variation que l'on connait dans les datations et les périodisations de la postmodernité, en littérature comme dans d'autres domaines, de même que la variété des définitions du concept de postmodernisme qui en découle, dépendent-elles très étroitement de l'échelle de grandeurs historiques qu'on prend en considération et du point de vue historique d'où l'on se place pour en parler.

John Barth, par exemple, dans son article de 1980, adopte le point de vue de l'écrivain des années cinquante et soixante, responsable, avec d'autres, de la mise en cause de la modernité

10 Ce n'est pas tant 1789 , en soi, qui représente une rupture dans l'histoire des formes sociales et économiques en France que la borne qu'elle incarne dans l'*aspectualité inchoative * - dirait le linguiste - qu'aura impliquée sa mise en discours dans des formations discursives particulières. 
littéraire jugée trop contraignante sur le plan cognitif et trop peu "démocratique" sur le plan sociopolitique ${ }^{11}$, pour situer l'émergence du postmodernisme romanesque autour de ces années qui ont vu naître en même temps que ses premières œuvres celles de ses confrères John Hawks, Robert Coover et autres William Gass; il place ensuite l'avènement de ces formes narratives postmodernes sur une échelle de grandeurs qui ne mesure que les changements esthétiques survenus dans l'histoire du roman depuis Cervantes et, plus particulièrement, depuis les débuts de la modernité, marqués par les œuvres de Kafka, Joyce et Proust, sans essayer de comparer, comme c'est le cas de nombreux théoriciens postmodernistes, les unités de mesure ainsi obtenues avec celles qui résulteraient d'une histoire des idées traitant des mutations scientifiques et philosophiques ou de l'histoire sociale appliquée aux grands changements de société. C'est ce point de vue, interne, et cette échelle, strictement esthétique et romanesque, qui fait du concept de postmodernisme chez Barth une notion qui relève essentiellement d'une "poétique d'auteur ", voire d'une *école littéraire" au sens restreint mais le plus noble de l'expression. En ce sens, sa conception rejoint celle de l'histoire de l'art, qui fonde sur des critères purement esthétiques, non sans rapport, toutefois, avec des jugements à saveur politique (sur l' "élitisme" du modernisme littéraire, chez Barth, ou sur l'uantihumanisme" du modernisme architectural, chez Bofill), la datation des débuts du postmodernisme et les périodisations diverses qui le composent ou en définissent l'héritage et la fortune. La notion de postmodernisme devient ainsi un concept à usage interne, propre à l'histoire littéraire ou à celle des arts, sans qu'une trompeuse homonymie ne l'amalgame abusivement aux enjeux des débats entre un Habermas, un Lyotard et un Rorty sur la postmodernité en philosophie ou des vives discussions entre sociologues et économistes déclenchées par les hypothèses d'un Fukuyama sur la "fin de l'histoire" qu'incarnerait l'âge dit postmoderne ou postindustriel.

D'autres points de vue, moins restreints, s'appliquant à des échelles de grandeurs qui embrassent à la fois notre conception

11 * Sans déchoir dans le simplisme moral ou artistique. le romancier postmoderne *aspire [...] à une fiction plus démocratique [sic] que ne le sont les merveilles de la dernière période du modernisme" (John Barth, op. cit., p. 403). 
du sujet et de la connaissance, voire nos ontologies, nos Weltanschauungen, nos épistémès, manifestent une plus grande ambition dans leur tentative de définition du postmodernisme et de description des objets historiques censés y correspondre. Ainsi le critère définissant la postmodernité par les incertitudes du "sujet" quant à son identité (sexuelle, ethnique, réelle ou fictive, individuelle ou fragmentée, etc.) - devenu l'un des passe-partout de la critique postmoderniste en littérature - ne peut en aucun cas caractériser la fiction dite postmoderne, dont les modes de représentation du sujet semblent au contraire s'appuyer sur le "personnage", au sens classique du terme, bien plus fermement qu'ils ne l'ont jamais été chez un Kafka ou chez un Joyce, dont les $\mathrm{K}$ et les Molly témoignent d'une mutation profonde du sujet, loin du rationalisme moderniste mis en cause par la pensée philosophique d'un Nietzsche et la théorie psychanalytique d'un Freud, qui leur sont à peu près contemporaines. La postmodernité philosophique, qui voit s'effondrer les Grands systèmes de l'Âge classique et des Lumières, de Descartes, Leibniz, Kant à Hegel, Fichte et l'Encyclopédie, pour donner lieu à ce que Steiner appellerait le commentaire infini de la pensée philosophique et de son histoire - dont témoigne le regard essentiellement philologique d'un Nietzsche sur les grands thèmes de la métaphysique occidentale, regard qu'empruntera à son tour Heidegger dans son exégèse des Grecs, de Kant, de Shelling et de Nietzsche lui-même cette postmodernité, dis-je, marque sans conteste une bifurcation importante dans la gnoséologie générale de la modernité philosophique, où les certitudes cognitives et perceptives du Sujet sont désormais hypothéquées par la double critique du rationalisme et de l'empirisme. Presque à la même époque, au tournant du siècle, les Grands systèmes romanesques des XVIII ${ }^{e}$ et XIX $^{e}$ siècles, des Mémoires et aventures d'un homme de qualité jusqu'aux RougonMacquart en passant par la Comédie bumaine, ont commencé à subir, de l'intérieur, une sérieuse mise en question, particulièrement sensible dans La recherche, Ulysse ou L'bomme sans qualités, dont les ambitieuses constructions n'aboutissent jamais au système ou à la synthèse, préférant tout dissoudre, y compris le sujet, dans une sorte d'analyse interminable, où chaque partie semble déborder le tout, l'élément embrasser l'ensemble. Les Grandes Synthèses poétiques, Atala, Jocelyn, La légende des siècles, subirent le même sort au travers des Chants de Maldoror, des Illuminations et du Coup de dés. Tout cela est bien connu, puisque la critique et l'histoire des idées datent déjà depuis 
plusieurs décennies l'avènement de notre modernité littéraire des grandes œuvres qui ont marqué le tournant de ce siècle, soit, à quelques exceptions près, de 1880 à 1920. Il semble ainsi qu'à la naissance d'une pensée postmoderne en philosophie - c'est-àdire d'une attitude résolument postcartésienne - corresponde l'émergence d'une esthétique moderne dans la littérature (et dans les Arts en général), où la critique du Réalisme fait pendant à celle qui a frappé le rationalisme classique. La critique littéraire postmoderniste, dans sa tentative d'établir des correspondances étroites entre les mutations épistémiques et les innovations esthétiques, n'a d'autres choix, dès lors, que de postdater les révolutions littéraires par rapport aux ruptures philosophiques auxquelles elles sont censées correspondre, ou de re-baptiser le modernisme littéraire émergeant dès la fin du siècle dernier en ayant recours à l'étiquette "postmoderne" déjà utilisée pour marquer la sortie hors du système métaphysique de la philosophie moderne qui aura sévi de Descartes à Hegel, avec quelques persistances chez Husserl et chez certains tenants de la philosophie analytique.

Dans tous les cas, la critique postmoderne est obligée à une révision de l'Histoire - qui n'est pas sans rappeler, comme je l'ai souligné au début, l'esthétique postmoderne de la déchronologisation de l'historicité propre aux Arts et aux Lettres, dont le "sens" est jugé "réversible". En voulant fonder ses tentatives de (re)périodisation littéraire sur des bases gnosélogiques et sur une histoire générale de la subjectivité, la critique postmoderne se condamne soit à l'anachronisme soit à une conception nominaliste de l'histoire. Dans le premier cas elle situe la révolution épistémique qu'aurait connue la pensée philosophique occidentale non plus par rapport à la fin des Grands systèmes (Nietzsche) et à l'avènement des sciences humaines (Marx, Freud, Saussure) mais par rapport au développement qu'a connu ce paradigme jusqu'à sa critique interne dans le cadre du poststructuralisme et, plus particulièrement, de la déconstruction, dont on sait pourtant qu'elle doit tout ou presque à ses prédécesseurs du tournant du siècle - ce qui lui permet de créer une certaine correspondance chronologique entre la fiction postmoderne apparue à la fin des années cinquante mais consolidée dans les années soixante-dix et quatre-vingt et la pensée poststructuraliste où s'enregistrent, dès la fin des années soixante, les ultimes mais décisives secousses du long séisme qui aura frappé l'épistêmè moderniste depuis plus d'un siècle. Dans le second cas, du nominalisme historique, l'historicité elle-même devient pure 
narrativité, les faits n'existent que nommés et racontés, le changement de noms et de récits entraînant automatiquement un changement dans les faits eux-mêmes - du coup, toute l'histoire de la modernité littéraire devient magiquement celle de sa postmodernité, le fil narratif de l'histoire littéraire enfin noué à celui de l'histoire générale des idées, qui forment ensemble une trame homogène, dont le destin, toutefois, n'est pas sans rappeler celui de la toile de Pénélope, détissée et retramée sans cesse, refaçonnée ici par les mots et par les récits, chaque fois nouveaux, qui changent continuellement le monde qu'ils décrivent et qu'ils racontent.

Cette double méprise historiographique, qui fait prendre les faits pour les fictions et inversement, est sans doute à l'origine des nombreux quiproquo qui alimentent les discussions entre critiques postmodernistes sur qui est et qui n'est pas postmoderne dans l'histoire récente et plus ancienne de la littérature universelle ou américaine: on a vu Cervantes et Sterne, leur Don Quichotte et leur Tristram Shandy sous le bras, joindre subrepticement les rangs des postmodernes, où se bousculent les auteurs états-uniens des deux ou trois dernières décennies, qui acceptent tant bien que mal de s'annexer par-dessus les frontières spatiales un Calvino, un Perec ou un Garcia-Marquez, voire une moitié de Duras ou un demi Robbe-Grillet, et par-delà les horizons temporels un Nabokov, un Borges ou un Faulkner quand ce n'est pas un tiers de Musil et un quart de Kafka, car la famille postmoderniste s'élargit sans cesse, véritable smala qui ne craint pas de s'adjoindre non seulement ses plus lointains cousins dans le monde contemporain mais ses ancêtres disparus qu'elle fait renaître à sa guise du plus profond de sa généalogie ${ }^{12}$. La conception postmoderne de l'histoire a une façon propre d'effacer le passé, qui est de le rendre actuel, Ulysse côtoyant Batman, Madonna et Diotima posant ensemble. Bref, le postmodernisme,

12 On a vu récemment paraître quelques livres de critiques postmodernistes sur la littérature québécoise et canadienne, qui nous ont appris que Nicole Brossard (celle d'Un livre et de Picture Theory) était postmoderne, tout comme Victor Lévy-Beaulieu, semble-t-il, et même Hubert Aquin et Maric-Claire Blais; doit-on en déduire que Félix Antoine Savard, Jean-Charles Harvey et Ringuet sont les auteurs * modernes *, voire * modernistes*, qui ont été dépassés par ces nouveaux promus à la postmodernité littéraire? 
malgré son nom, ne supporte pas le deuil, la mort: il veut tout vivre dans l'espace, dans la présence. Il n'est qu'une vaste mémoire dans laquelle tout, à tout moment, peut être rappelé, sans distinction de dates, d'antériorité et de postériorité; sa mémoire historique est une "banque de données" à laquelle n'importe quelle "requête" peut être "adressée", comme on dit dans les langages "branchés", et cela sans qu'aucun ordre, autre que vaguement esthétique, ne structure ces myriades de data proprement mises en "banque", d'où on les ressortira bientôt avec les intérêts qu'elles rapportent.

Je caricature, bien sûr, non sans quelque mauvaise foi. Mais l'histoire n'est pas faite de data et l'historicité n'a rien de banquaire: le passé existe, dirait Heidegger, et nul présent ne le rendra à la présence, pas même ce temps hors temps qu'est la temporalité soi-disant postmoderne. On ne peut rester indifférent à une conception de l'histoire littéraire qui fait correspondre l'un de ses moments, le "moment postmoderne ", tantôt à la fin même de l'histoire, tantôt à un prolongement de l'époque moderne, tantôt encore à une réaction face à cette dernière suivie d'un retour à l'une ou à l'autre des périodes dites prémodernes, tantôt enfin à une synthèse, souhaitée par Barth ${ }^{13}$, de la modernité et de la prémodernité, quand ce n'est pas le plus souvent à un dépassement encore insaisissable de tous les moments qui l'ont précédé. Je ne cherche pas à nier l'existence d'un mouvement esthétique auquel on puisse accoler l'étiquette "postmoderniste", comme on a jadis donné à un certain type de récits le label "Nouveau roman", qui n'a plus rien de nouveau aujourd'hui, ou comme on a appliqué naguère à une certaine école poétique l'appellation "futuriste", devenue bientôt passéiste, mais le préfixe post ne doit pas plus prêter à illusions que l'adjectif Nouveau ou le morphème néo-: le postmodernisme va mourir, meurt, est mort, d'autres tendances esthétiques le remplaceront, l'ont remplacé, et d'autres étiquettes viendront bientôt, dans dix ans et dans un siècle, se superposer au sien et sans doute s'y substituer pour désigner les mêmes mutations formelles et thématiques, vues sous un autre angle et par rapport à une échelle historique différente, c'est-à-dire selon un aspect qui en révèlera certaines propriétés que seuls les enjeux historiques des temps à venir pourront faire surgir.

13 John Barth, op. cit., p. 403. 
Le postmodernisme nous a confirmé que l'histoire n'est pas immuable: le passé vit, changeant de forme et de sens, au gré des changements de cap où notre avenir se dessine. Ce sera là son apport à la pensée contemporaine. Mais faut-il en conclure que le temps n'existe que dans l' "après coup" de sa désignation par une étiquette ou une "catégorie" historique qui le date et le périodise? Qu'aucune temporalité n'échappe à ce "moment d'après" qu'est sa narrativation ou sa discursivation, soit sa prise en charge par le langage propre à l'histoire, qui lui-même change avec le temps? L'Histoire se revisite, bien sûr, mais au risque de maints "révisionnismes", qui muent le passé en un éternel présent, où l'on ne supporte pas de voir érigés dans la mémoire les obstacles qui empêchent d'élaborer tel ou tel projet d'avenir. L'aplatissement du temps guette la pensée postmoderniste, dont la double obsession consiste à ontologiser l'histoire, devenue collection de faits tous réels au même titre et appartenant de même façon à son univers de référence, ou à nominaliser la pensée historique, transformée en collections de mots et de récits qui renomment sans relâche le monde quasi fictif dont elle fait son objet. Patchwork de faits, de dates, casse-tête de mots, de récits, l'histoire et le discours historique propres à la pensée postmoderne semblent avoir perdu, d'une part, toute transcendance et, d'autre part, toute intentionnalité, en ce sens que le temps n'y est plus défini en son extériorité, et que la parole qui le désigne ne possède plus de véritable objet intentionnel, qu'elle puisse viser en-dehors d'elle. L'aspectualité du discours historique, qui voit les faits depuis un certain point de vue et à l'intérieur d'une certaine échelle de grandeurs, ne remet nullement en cause l'extériorité et l'hétérogénéité radicale des faits par rapport à leur mise en discours; elle dit simplement le rôle de la perception sémantique ou discursive dans l'élaboration des catégories historiques, dont l'objet continue d'exister, fondamentalement, hors de l'acte de perception proprement dit et, $a$ fortiori, hors de l'acte d'énonciation du sujet qui le prend en charge dans le langage, de la dénomination à la narrativation. $\mathrm{Si}$ le relecture postmoderniste de l'histoire - de celle, notamment, de la littérature - , peut avoir un certain avenir dans les sciences actuelles de l'interprétation, comme dit Gadamer, c'est grâce à un recours à une plus grande réflexivité, qui l'amène à voir et à revoir ses propres processus d'aspectualisation du procès 
130

historique, ainsi qu'à les montrer et à les expliciter, de sorte que l'ambiguité du projet par rapport à ses possibles visées soit rapidement levée.

Ou bien le postmodernisme, en théorie de la littérature, consiste à décrire un mouvement littéraire dans ses diverses composantes, esthétiques, sémiotiques, thématiques, éthiques et sociopolitiques - dans lequel cas il doit circonscrire son objet dans le cadre d'une ou plusieurs littératures nationales, clairement identifiées, et d'une période historique relativement homogène, avec son moment d'émergence et son possible déclin; ou bien il vise de manière plus ambitieuse à réexaminer le programme entier de la modernité littéraire, aux deux sens du terme (depuis la Renaissance, par rapport au Moyen-Âge et à l'Antiquité, et depuis les premières Avant-gardes postsymbolistes, par rapport au Romantisme et au Classissisme), à la lumière des changements épistémiques et socio-économiques qui ont marqué l'ensemble des formations sociales et discursives des derniers siècles - dans lequel cas, évidemment, les quelques tentatives de repériodisation qui ont été faites jusqu'à maintenant ne peuvent aucunement suffire. L'échelle de grandeurs n'est pas la même dans l'un et l'autre cas, et c'est l'une des maladresses les plus constantes de la critique postmoderniste de les confondre ou de les entremêler, mesurant les grandes ruptures épistémologiques à l'aune du dernier roman d'une Kathy Acker ou les caractéristiques formelles de l'œuvre d'un Vonnegut en faisant appel à la Critique du rationalisme philosophique. Si une telle commune mesure doit être prise entre les mutations esthétiques du discours romanesque et les changements cognitifs majeurs qu'a connus la pensée philosophique depuis plus d'un siècle, il faut prendre les choses de plus haut, et réinterroger sans préjugé d'école comme sans $a$ priori pour une littérature particulière (américaine ou autre, récente ou plus ancienne), les paramètres et les variables qui permettent de parler d'une métamorphose sensible à la fois dans les systèmes de pensée et dans les formes littéraires, remettant ainsi en question les distinctions entre modernité et postmodernité littéraires en fonction d'unités de mesure plus larges, propre à l'histoire longue des épistémès, des doxa, des "visions du monde", dont les changements sont beaucoup plus rares et lents que ceux qui surviennent à chaque décennie dans les différentes littératures. On aura alors su faire la distinction entre un mouvement littéraire postmoderniste plus ou moins éphémère, en tout 
cas ponctuel, qui a son ancrage institutionnel dans un moment socio-historique et dans un lieu sociopolitique précis, et un mouvement de fond certainement plus important où la littérature occidentale a su accompagner, depuis plus d'un siècle, les grandes mutations de la pensée et des formes que la société dite moderne s'est données. La périodisation de ce dernier mouvement ne supporte d'autre mesure qu'à grande échelle, où les distinctions entre modernism, late modernism, postmodernism pratiquées par une certaine critique actuelle ne sont d'aucun secours réel. Récrivons l'histoire, oui, nous n'en avons pas le choix, mais sachons laquelle: ou bien l'histoire de la pensée telle qu'elle s'exprime dans les formes littéraires depuis plus d'un siècle, ou bien celle d'un mouvement littéraire anglo-américain qui a essaimé dans un certain nombre de cultures depuis deux ou trois décennies. La notion de postmodernité varie d'une histoire à l'autre, et cette variation doit s'exposer ouvertement si l'on veut conserver à l'Histoire son véritable feuilleté et ne pas l'aplatir en rabattant l'une sur l'autre les différentes histoires qui la composent, chacune ayant son échelle propre. 